Subscriber access provided by Caltech Library Services

\title{
Communication
}

\section{Superstrength through Nanotwinning}

Qi An, William A. Goddard, Kelvin Y Xie, Gi-Dong Sim, Kevin J. Hemker, Tyler Munhollon, Muhammet Fatih Toksoy, and Richard A. Haber

Nano Lett., Just Accepted Manuscript • DOI: 10.1021/acs.nanolett.6b03414 • Publication Date (Web): 07 Nov 2016

Downloaded from http://pubs.acs.org on November 9, 2016

\section{Just Accepted}

"Just Accepted" manuscripts have been peer-reviewed and accepted for publication. They are posted online prior to technical editing, formatting for publication and author proofing. The American Chemical Society provides "Just Accepted" as a free service to the research community to expedite the dissemination of scientific material as soon as possible after acceptance. "Just Accepted" manuscripts appear in full in PDF format accompanied by an HTML abstract. "Just Accepted" manuscripts have been fully peer reviewed, but should not be considered the official version of record. They are accessible to all readers and citable by the Digital Object Identifier (DOI®). "Just Accepted" is an optional service offered to authors. Therefore, the "Just Accepted" Web site may not include all articles that will be published in the journal. After a manuscript is technically edited and formatted, it will be removed from the "Just Accepted" Web site and published as an ASAP article. Note that technical editing may introduce minor changes to the manuscript text and/or graphics which could affect content, and all legal disclaimers and ethical guidelines that apply to the journal pertain. ACS cannot be held responsible for errors or consequences arising from the use of information contained in these "Just Accepted" manuscripts. 


\title{
Superstrength through Nanotwinning
}

\author{
Qi An, ${ }^{1}$ William A. Goddard III ${ }^{*}$, Kelvin Y. Xie, ${ }^{2}$ Gi-dong Sim, ${ }^{2}$ Kevin J. Hemker ${ }^{2}$, Tyler \\ Munhollon, ${ }^{3}$ M. Fatih Toksoy, ${ }^{3}$ and Richard A. Haber ${ }^{3}$ \\ ${ }^{1}$ Materials and Process Simulation Center, \\ California Institute of Technology, Pasadena, CA 91125, U. S. A. \\ ${ }^{2}$ Department of Mechanical Engineering, Johns Hopkins University, Baltimore, MD, 21218, USA \\ ${ }^{3}$ Department of Materials Science and Engineering, Rutgers, \\ The State University of New Jersey, Piscataway, NJ 08854 \\ *Corresponding author E-mail: wag@wag.caltech.edu
}
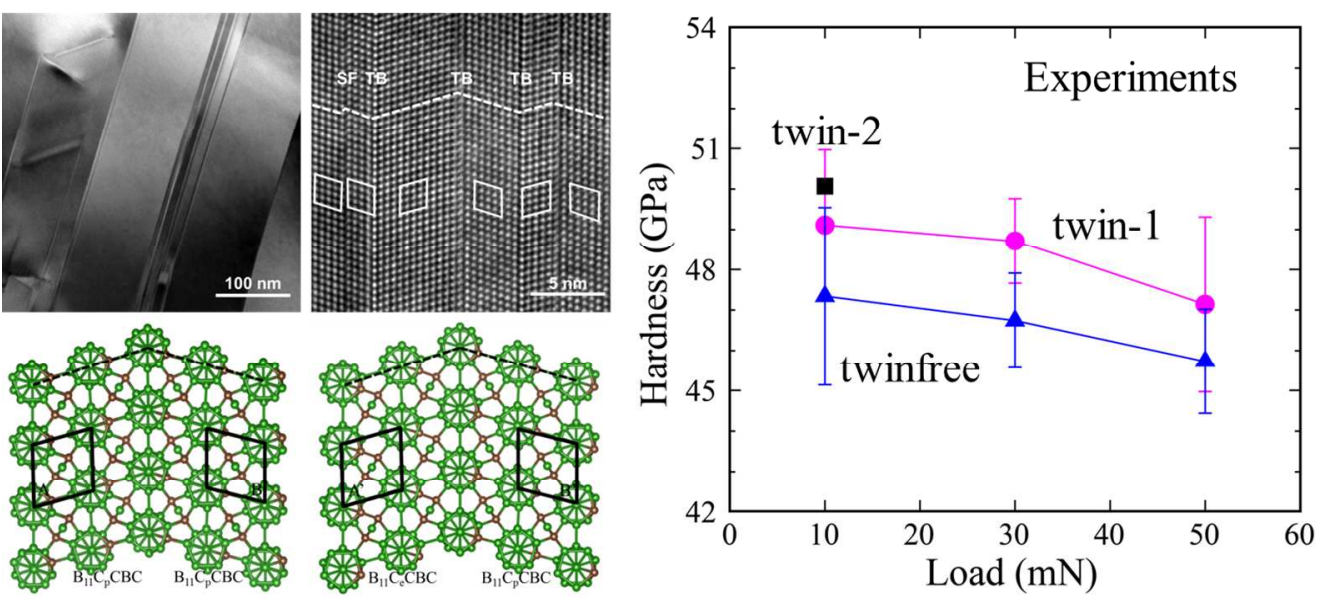

TOC Figure

\begin{abstract}
The theoretical strength of a material is the minimum stress to deform or fracture the perfect single crystal material that has no defects. This theoretical strength is considered as an upper bound on the attainable strength for a real crystal. In contradiction to this expectation, we use quantum mechanics $(\mathrm{QM})$ simulations to show that for the boron carbide $\left(\mathrm{B}_{4} \mathrm{C}\right)$ hard ceramic, this theoretical shear strength can be exceeded by $11 \%$ by imposing nano-scale twins. We also predict from $\mathrm{QM}$ that the indentation strength of nano-twinned $\mathrm{B}_{4} \mathrm{C}$ is $12 \%$ higher than that of the perfect crystal. Further we validate this effect experimentally, showing that nano-twinned samples are harder by $2.3 \%$ than the twin-free counterpart of $\mathrm{B}_{4} \mathrm{C}$. The origin of this strengthening mechanism is suppression of twin boundary (TB) slip within the nano-twins due to the directional nature of covalent bonds at the TB.
\end{abstract}

Keywords: Superhard ceramics, Hardness, DFT, Nanoindentation, Deformation mechanism. 
The ideal strength of a material is the maximum stress above which elastic strain is unstabile. $^{1,2}$ This is a fundamental mechanical property directly related to the nature of chemical bonding in the crystal. ${ }^{3}$ Early attempts to compute the ideal strength from atomic models go back to Frenkel ${ }^{4}$ and Orowon ${ }^{5}$, but now quantum mechanics (QM) can be used to calculate this limit. Of course, the measured yield strength in real crystals is expected to be much lower than the ideal strength because of the presence of defects (mobile dislocations, grain boundaries, cracks). Recently ultra-strength phenomena have been observed in nanocrystals ${ }^{6}$ and nanopillars ${ }^{7}$, raising the question of how close one can get to the intrinsic upper bound, the ideal strength. Although this ideal strength cannot be measured accurately in experiments, we can use well-established QM approaches to calculate it. ${ }^{8}$

It is well known that the strength of metal alloys often increases as the average crystallite (grain) size decreases due to the impedance of dislocation motion by grain boundaries, a phenomena referred to as the Hall-Petch relationship. ${ }^{9,10}$ However, when the grain size decreases below a critical value, grain boundary migration ${ }^{11}$ or sliding ${ }^{12}$ dominates the deformation mechanism rather than dislocation motion, leading to a decrease in the strength as the grain size decreases further. These relationships are widely applicable to crystalline metals where mobile dislocations play essential roles in plastic deformation. ${ }^{13,14}$ However, dislocations in ceramics are often sessile because of the rigid structures arising from the covalent or ionic bonding (except at extremely high temperatures). Thus, understanding of the Hall-Petch relationships in ceramics remains under study. ${ }^{15,16}$ 
Grain boundaries (GBs) and interfaces in most crystalline solids are characterized by an increased energy of formation, representing the weak links compared to perfect crystals. ${ }^{17,18}$ Only coherent interfaces with small lattice mismatch, such as twin boundaries (TBs), can reach a strength approaching that of the perfect crystal, but never exceeds it. ${ }^{19}$ TBs are energetically more stable than conventional GBs, leading to increased strengthening compared to conventional GBs. ${ }^{20}$ Recent experiments observed that nano-twinned cubic boron nitride and diamond with very fine twin thickness down to several nanometers dramatically increased the strength and hardness, compared with the effect of grain boundaries usually been observed in nanocrystalline metals. $^{21,22}$ This raised the question of how close to the intrinsic strength of a covalent solid one can get with nanoscale twins.

We consider here boron carbide $\left(\mathrm{B}_{4} \mathrm{C}\right)$ as our prototypical hard ceramic. $\mathrm{B}_{4} \mathrm{C}$ exhibits important properties such as thermal stability, high hardness, abrasion resistance, and low density, making it a candidate for such applications as abrasive powders, body armor, and neutron radiation absorbents. ${ }^{23,24}$ In particular, the low density of $\sim 2.52 \mathrm{~g} / \mathrm{cm}^{3}$ makes it very attractive for body armor applications. ${ }^{25}$ However, the brittle failure in $\mathrm{B}_{4} \mathrm{C}$ arising from amorphous band formation under modest impact has impeded wider insertion of $\mathrm{B}_{4} \mathrm{C}$ into extended engineering applications. ${ }^{26-28}$ In addition, mobile dislocations are rarely observed in $\mathrm{B}_{4} \mathrm{C}$, especially at low temperature. ${ }^{29}$ Thus, we focus on the effects of nanotwinning.

In this paper, we apply QM (density functional theory (DFT) at the level of the PerdewBurke-Ernzerhof (PBE) functional) to determine the stress-strain relationships, the ideal shear 
strength, and the failure mechanism for nano-twinned boron carbide under both pure shear deformation and biaxial shear deformation. We show from QM that the intrinsic strength for nano-twinned structures of $\mathbf{B}_{\mathbf{4}} \mathbf{C}$ is higher than the strength limit of the perfect crystal and that this strengthening effect depends on the twin densities. We validate these QM predictions through nanoindentation experiments on both twinned and twin-free $\mathrm{B}_{4} \mathrm{C}$ samples. The QM studies show that this extraordinary strengthening of the nano-twinned system originates from highly anisotropic shear stress response in nano-twinned $\mathrm{B}_{4} \mathrm{C}$ due to directional bonding configuration at the TBs. This provides an accessible mechanism by which to surpass the strength limit of covalent solids.

Although ideal strength is defined by elastic instability, its precise definition is somewhat subtle. The ideal strength depends on the geometry of the load. Here we define the ideal limiting shear stress as the lowest value among all possible slip systems. ${ }^{28}$ Our previous DFT study ${ }^{28}$ on 11 possible slip systems for crystalline $\mathrm{B}_{4} \mathrm{C}$ showed that this limit is for shear along $(01 \overline{1} \overline{1}) /<\overline{1} 101>$, which leads to the amorphous band formation along the $(01 \overline{1} \overline{1})$ plane found experimentally. ${ }^{27}$ We showed that this failure mechanism involves two-steps: (1) First, the B-C bond connecting two icosahedra breaks, leading to formation of a reactive carbene; (2) Then this negative carbene reacts with the positive middle boron in the C-B-C chain as it is sheared past the carbene, breaking the bonds within the icosahedron. ${ }^{28}$

Our density functional theory (DFT) calculations were performed with the VASP package, ${ }^{30-32}$ using the PBE functional and the projector augmented wave method to account for 
the core-valence interactions. ${ }^{33}$ To examine the pure shear deformation of the twin structures, we imposed the strain for a particular shear plane while allowing full structural relaxation of the atoms for the other five strain components. ${ }^{34}$ To simulate the hardness measurement, we applied biaxial stress shear deformation methods on the nanotwinned structure that mimics the indentation conditions. ${ }^{35,36}$ The simulation details are in the Supplemental Information (SI).

Previous studies have shown ${ }^{37}$ that high twin densities can be introduced experimentally into $\mathrm{B}_{4} \mathrm{C}$, with an example shown in Fig. 1(a,b). Close examinations of these materials showed that both symmetric twins and asymmetric twins can be present in highly twinned samples. ${ }^{23,37}$ Our QM models (Fig. 1(c,d)) show that the "asymmetric twins" are actually phase boundaries between the ground state structure $\left(\mathrm{B}_{11} \mathrm{C}_{\mathrm{p}}\right)(\mathrm{CBC})$ and the $\left(\mathrm{B}_{11} \mathrm{C}_{\mathrm{e}}\right)(\mathrm{CBC})$ higher energy structure.$^{37}$ Our QM simulations of the optimized bulk symmetric twin structure contains 120 atoms with cell parameters of $\mathrm{a}=10.416 \AA, \mathrm{b}=5.208 \AA, \mathrm{c}=17.727 \AA, \alpha=90.0^{\circ}, \beta=90.0^{\circ}, \gamma=$ $65.3^{\circ}$ leading to a density of $2.521 \mathrm{~g} / \mathrm{cm}^{3}$. For the asymmetric twin, the QM cell parameters are a $=10.411 \AA, \mathrm{b}=5.205 \AA, \mathrm{c}=17.798 \AA, \alpha=90.9^{\circ}, \beta=89.1^{\circ}, \gamma=65.0^{\circ}$ with a density of 2.519 $\mathrm{g} / \mathrm{cm}^{3}$. The twin boundary (TB) is along the "a" direction leading to 2-layer crystalline structures between TBs along the "c" direction, as shown in Fig. 1. We also constructed the 3-layer twinned model with 180 atoms and 4-layer twinned model with 240 atoms to examine the effects of multiple twin layer.

To determine the plausible slip system in these twin structures, we considered two cases:

(1) one with shear parallel to the TB plane, $(01 \overline{1} \overline{1})$, which is also the plane for forming the 
amorphous band on both QM and experiment;

(2) the other with shear along a plane perpendicular to the TB plane.

These models of shearing and the QM stress-strain curves are displayed in Fig. S1 of the SI, showing that the maximum ideal shear stress along the TB plane is $43.6 \mathrm{GPa}$, which is $5.2 \mathrm{GPa}$ lower than for shear perpendicular to the TB plane. This indicates that shear along the TB plane is more favorable, so we now focus on shear deformation along the TB plane.

To investigative the effect of twins on crystal strength, we sheared both systems (with both symmetric and $t$ asymmetric TB), leading to the stress-strain relationships shown in Fig 2(a). The maximum ideal shear stress for symmetric twin is $43.6 \mathrm{GPa}$, which is $1.4 \mathrm{GPa}$ larger than for the asymmetric twin and $4.7 \mathrm{GPa}(11 \%)$ larger than for the perfect crystal. Thus, the strength of both twin structures predicted from QM exceeds the theoretical ideal shear strength of the perfect crystal by $10 \%$, even though the twin structures are thermodynamically less stable than the perfect crystal.

This $10 \%$ higher strength for the twinned structure compared to the perfect crystal is found even though the shear moduli (the slope of stress-strain curve) of the nano-twinned structures are smaller than the perfect crystal (because of the interfacial energy). This is in stark contrast to the common intuition that high modulus and high hardness go together. The reason for this anti-intuitive result is that for this symmetric TB, the lower half shears along the $<\overline{1} 101>$ direction, as shown in Fig. 2(b), while the upper half part shears along the opposite direction of $<1 \overline{1} 0 \overline{1}>$, which requires higher stress than shear along $<\overline{1} 101>$. This is seen from the shear 
deformation of the perfect crystal along these two directions shown in Fig. S2 of the SI. This differential in the ideal shear stresses for shear along two opposite directions in the same slip plane arises from the directional nature of the covalent bonding. For the twinned structures, the higher shear on the upper half part prevents the shear on the lower half, increasing the ideal shear stress. Since hardness is related to the resistance to plastic deformation, we expect and find that both symmetric and asymmetric TBs increase the hardness, but the symmetric TBs increase it more than asymmetric TBs.

For symmetric twins, the failure process (see Fig. 2(b-d)) exhibits the same two-step process as for the single crystal.

(1) Firstly, as the strain increases from 0 to 0.209 , the structure experiences elastic deformation. The stretching of B-C bonds between icosahedra in the lower half part is $43.9 \%$ larger than in the upper part because the lower part is deformed along the easy direction. This distorts the B-C bonds between icosahedra in the lower part pushing them toward the breaking point, as shown in Fig. 2(b). In contrast to the perfect crystal, where the shear stress first drops and then increases, ${ }^{28}$ the shear stress for the twin case increases continuously because the bonds in upper half part remain intact.

(2) As the strain increases to 0.322 , the carbon-boron bond between icosahedra breaks, forming a spin singlet carbene lone pair on the $\mathrm{C}$ of the icosahedron in the TB plane, as shown in Fig. 1(c). This is illustrated in the enlarged figure in Fig.2(c), which shows the electron localization function (ELF). ${ }^{38}$ Starting at 0.322 strain and removing the stress leads recovers exactly the 
starting structure. The broken B-C bond between the icosahedra simply reforms during the geometry optimization. Thus, the structure has not failed yet at 0.322 strain. Passing the critical stain of 0.322 , the middle boron atom of the $\mathrm{C}-\mathrm{B}-\mathrm{C}$ chain reacts with the carbene lone pair in the TB plane, breaking the C-B-C chain and partially breaking the icosahedra, as shown in Fig. 2(d), releasing the stress. This failure process partially breaks only one layer of icosahedra in the TB plane, while relaxing the other icosahedra back to their original intact status.

To examine how the multiple layers between TBs might affect the deformation mechanism described above, we constructed the 3-layer and 4-layer twin structures shown in Fig. S3(a,b) of the SI and applied pure shear deformation to them. The stress-strain relationships for various twinned structure in Fig. S3(c) show that the maximum shear stress decreases from $43.6 \mathrm{GPa}$ to 41.2 and 40.9 GPa as the number of layers between TBs increases from 2 to 3 and 4, indicating a weakening effect for multiple layer twinned structures.

The structural changes for this 3-layer twinned structure under pure shear deformation are displayed in Fig. S4(a, b) of SI. As the shear strain increases to 0.322, which corresponds to the maximum shear stress, the B-B bonds between icosahedra (within the region of the easy shear direction) break. As the shear strain increases to 0.345 , the icosahedra within the twin layer and the layer next to the TBs deconstruct due to the interaction of $\mathrm{C}-\mathrm{B}-\mathrm{C}$ chain and the $\mathrm{B}_{11} \mathrm{C}$ icosahedra.

Although the twinned structure has higher ideal shear stress than perfect crystal, the failure occurs along the TBs in the 3 or 4 layer twinned structure because the high interfacial energy 
concentrates the shear strain within the TB regions. The preference for failure within TB regions plays an important role in strengthening the twinned structure with multiple layers. However, this strengthening effect would likely decrease when the TBs are too separated. Thus, we expect that the strengthening effects from twins would be greatest when their separations are at the nanoscale.

The detailed failure mechanism for asymmetric twins is described in the SI (Fig. S5), which after failure, leads to partially broken icosahedra with a density of $2.480 \mathrm{~g} / \mathrm{cm}^{3}$.

Our large scale $(\sim 200,000$ atoms/cell) ReaxFF reactive dynamics simulations reported previously $^{39}$ starting with a single crystal showed that brittle failure arises due to the $5.9 \%$ increased density within the amorphous band compared to the crystal. This increased density in the amorphous band leads to tension that eventually results in cavitation and then crack formation. ${ }^{39}$ It will be interesting to examine the how nanoscale twins affect crack propagation and the interaction between a crack and twin boundaries in the future.

To validate our QM predictions of improved intrinsic strength for the nano-twinned boron carbide, we performed nanoindentation experiments (10 indents for each loading condition on each sample) on twinned and twin-free boron carbide samples. We fabricated two highly twinned samples (twin-1 and twin-2) and obtained the twin-free counterpart from Coosteck. ${ }^{40}$ The detailed processing information is provided in the SI. All samples possess $\mathrm{B}_{4} \mathrm{C}$ chemical stoichiometry and have most grains $5-15 \mu \mathrm{m}$ in size, as shown in the TEM images in Fig. S6 of SI. X-ray diffraction (XRD) scans showed no appreciable amount of second phases in all 
samples. Twin-free and twin-1 samples are fully dense, while twin-2 sample is $\sim 90 \%$ dense. For the twin-free sample, $\sim 95 \%$ of the grains were found to be single crystalline. On the other hand, in both twinned samples, all grains contain twins. Measurements of 200 twins in each twinned sample revealed that twin-2 has higher twin density than twin-1 (Fig. 3(a)). For example, twins less than 1um in size constitute only $20 \%$ area of twin- 1 sample, but occupies $80 \%$ area in twin-2 sample. Note that the twin density is not uniform even within one grain. Twin spacing can range from a few atomic planes to $\sim 10 \mu \mathrm{m}$. The cumulative area twin density plots (Fig. 3(a)) provide quantitative microstructural information of both twinned samples, which will be used to elucidate the hardness observations.

The hardness values obtained from nano-indentation tests show that the twinned sample is consistently harder than the twin-free sample (Fig. 3(b)). Under $10 \mathrm{mN}$ load, the hardness of twin-2 $(50.07 \mathrm{GPa})$ and twin-1 $(49.11 \mathrm{GPa})$ are $4.2 \%$ and $2.3 \%$ higher than that of twin-free sample (48.03), respectively. It is noteworthy twin-2 is also harder than twin- 1 . This can be attributed to the higher twin density in twin-2 compared to twin-1 (Fig. 3(a)). This observation agrees with our prediction that higher twin density leads to improved hardness and strength. To establish that the twinned samples are statistically significantly harder than the twin-free sample, we carried out the Student's t-test, leading to confidence intervals of $83 \%$ for the twin- 1 sample and $97 \%$ for twin- 2 sample. Note that the twin- 2 sample is $90 \%$ dense, and the effect of porosity became apparent when larger loads were applied (e.g. 30 and $50 \mathrm{mN}$ ). Regardless, when interrogating the dense specimens, the twin-1 sample was observed to be consistently harder than 
the twin-free sample under all loading conditions (Fig. 3b).

We note that other microstructural features such as grain boundaries, stoichiometry and second phases may also affect the experimental hardness values. Regarding grain boundaries, the samples we used in this study (both highly twinned and twin-free) have grain sizes ranging from 5-15 $\mu \mathrm{m}$. In nanoindenation tests, the measured indent depth ranged from 80 to $275 \mathrm{~nm}$. The diagonal indentation length under $10 \mathrm{mN}$ load is only 0.85 micron - much smaller than the boron carbide grains. Thus, most indents should be within the grains. Moreover, 10 indents were performed for each loading condition on each sample, and even when grain boundaries were probed, their effect should be averaged out. Regarding stoichiometry and second phases, XRD results showed all samples have $\mathrm{B}_{4} \mathrm{C}$ stoichiometry and no appreciable amount of second phases were present, thus their effects can be precluded. Therefore, the improved hardness can be attributed to the presence of high density twins in the microstructure. Taken together, the experimental observations demonstrate that twinned samples are harder than twin-free samples, in good agreement with our QM predictions - superstrength through nanotwinning. Although our experiments agree very well with QM predictions, the significant difference between twin spacing in QM and experiments make it complex to interpret the experimental observations. In the experimental conditions, some other deformation mechanisms (such as defect nucleation, crack initiation) might play a role in the strengthening mechanism in addition to nanotwinning.

The stress conditions in indentation experiments are complex compared to the pure shear deformation. To determine the mechanism underlying this increased strength of the twinned 
structures under indentation, we also used QM to apply biaxial stress shear deformation to the nano-twinned structures aimed at mimicking indentation experiments, and compared with the perfect crystal.

The predicted shear-stress-shear-strain relationships for biaxial stress shear deformation are shown in Fig. 4. The perfect crystal experiences non-linear deformation for shear strains ranging from 0.136 to 0.263 , before mechanical failure. The shear stress decreases initially and then increases in this deformation region, which is similar to our previous results for pure shear deformation. ${ }^{28}$ However, for the nano-twinned structures, the stress increases continuously and suddenly drops without obvious non-linear deformation. The maximum shear stress for the symmetric 2-layer twinned structure is $31.8 \mathrm{GPa}$ compared to $31.3 \mathrm{GPa}$ for the asymmetric 2-layer twinned structure. These both exceed the strength limit of the perfect crystal (28.5 GPa) by $11.6 \%$ for the symmetric twin and $9.8 \%$ for the asymmetric twin. The maximum shear stresses for 3-layer and 4-layer twinned structures decrease to 30.0 and $29.9 \mathrm{GPa}$, which are $5.9 \%$ and 5.6\% higher than the perfect crystal, respectively. This predicted increase in strength by $10.7 \%, 5.9 \%$ and $5.6 \%$ for the 2-layer, 3-layer and 4-layer twinned structures, respectively, agrees with our experimental observations that twinned structures are stronger than twin-free structures and that high twin density leads to more pronounced strengthen effects.

To understand the mechanism underlying this nanotwin strengthening, we examined the structural changes as shown in Fig. 5(a-h). For the perfect crystal, the C-B-C chain bends as biaxial stress is applied, putting the $\mathrm{B}^{+}$at the middle of the chain close to the $\left(\mathrm{B}_{11} \mathrm{C}_{\mathrm{p}}\right)^{1-}$ 
icosahedron. The C-B-C angle bends to $158.5^{\circ}$ as the strain increases to 0.136 , as shown in Fig. 5(a). After the strain increases further to 0.155 , the C-B-C chain starts to bend, changing from $180^{\circ}$ to $134.7^{\circ}$, as the system deforms plastically for strains of 0.155 to 0.263 (Fig. $5(\mathrm{~b})$ ). Then at 0.263 strain the middle B interacts with the cage carbene as shown in Fig. 5(c); destabilizing the skeletal bonding in the icosahedron. Finally, this leads to the cage decomposition and mechanical failure at 0.297 strain, as shown in Fig. 5(d).

For the symmetric twin under biaxial stress conditions, the deformation process shows only a one step failure process. As the strain increases to 0.187 , the C-B-C chains near the TB plane bend to $141.0^{\circ}$ at which point the middle boron atom starts to interact with the icosahedron in the TB plane, as shown in Fig. 5(e). The C-B-C chains in the other layers also bend, but much less than those near the TB plane because the upper half part shears along the hard direction. At 0.209 strain this leads to breaking one layer of icosahedra in the TB plane, as shown in Fig. 5(f).

The asymmetric twin system shows a character similar to the symmetric twin. The C-B-C chains near the TB bend to $142.2^{\circ}$ before failure, as shown in Fig. $5(\mathrm{~g})$ for 0.173 strain. The middle boron in the bent $\mathrm{C}-\mathrm{B}-\mathrm{C}$ chains interacts with the icosahedra under the biaxial stress state, leading to breaking of the icosahedra in the TBs as the strain increases to 0.191, as shown in Fig. $5(h)$.

Although the icosahedra in the twinned structures break at a smaller strain than in the perfect crystal, the plastic deformation in the twin structures leads to a continuously increasing shear stress. This leads to a maximum shear stress $11.6 \%$ larger for symmetric twin and $9.8 \%$ 
larger for asymmetric twin than the strength limit for the perfect crystal, strengthening the twinned materials.

Fig. 4 shows that the non-linear regime appears to be more affected by biaxial loading in nano-twinned structure than the perfect crystal. This non-linear behavior arises from the deformation mechanism as shown in Fig. 5. For pure shear deformation, the broken B-C bond between icosahedra leads to this non-linear behavior. In biaxial shear deformation, but the concomitant compression greatly reduces the non-linear behavior because the B-C bond between icosahedra is prevented from breaking.

Since the experimental twinned samples have large twin spacing variation and some regions have much lower twin densities than the QM model, the experimental strengthening effect is only $2.3 \%$ for the twin sample.

In summary, we combined QM simulations and nanoindentation experiments to demonstrate that nano-twinned $\mathrm{B}_{4} \mathrm{C}$ has improved intrinsic strength compared to the perfect crystal, even exceeding the ideal strength. The strengthening mechanism is suppression of TB slip due to the directional nature of covalent bonds. These studies indicate that the concept of theoretical shear strength for strong covalent solids must be re-examined for nanoscale planar defects. These studies also suggest that stronger boron carbide might be developed by utilizing nanoscale twins.

\section{ASSOCIATED CONTENT}

\section{Supporting Information}


Supplemental information includes the DFT simulation details; The failure mechanism of asymmetric twins; The detailed deformation mechanism of perfect crystal and nano-twinned structures under biaxial stress conditions; Material fabrication and microstructural characterization; The nano-indentation details. Figures include the shear model and stress-strain relationship for the symmetric twins shearing along TB plane and perpendicular to TB plane; The stress-strain relationship for perfect crystal shearing along two opposite directions in the same slip plane of $(01 \overline{1} \overline{1})$; Multiple layer twinned structure and the stress-strain relationships for twinned and perfect $\mathrm{B}_{4} \mathrm{C}$ under pure shear deformation; The structural changes for the 3-layer twinned structure under pure shear deformation; The structures and ELF of asymmetric twin under pure shear deformation; The TEM image of twinned and twin-free samples; This material is available free of charge via the Internet at http://pubs.acs.org.

\section{Acknowledgements}

Q.A. and W.A.G. received support from the Defense Advanced Research Projects Agency (W31P4Q-13-1-0010, program manager, John Paschkewitz), the Army Research Laboratory (W911NF-12-2-0022), and the National Science Foundation (DMR-1436985). K.X and K.J.H. acknowledge support from the Army Research Laboratory (W911NF-12-2-0022). T.M., F.M.T and R.A.H acknowledge support from the Army Research Laboratory (W911NF-12-2-0022). We thank Tomoko Sano at ARL for providing us the twin-2 sample.

\section{Additional information}

Notes 
The authors declare no competing financial interests.

1

2

4

5

6

7

8

9

10

11

12

13

14

15

16

17

18

19

20

21

22

23

24

25

26

27

28

29

30

31

32

33

34

35

36

37

38

39

40

41

42

43

44

45

46

47

48

49

50

51

52

53

54

55

56

57

58

59

60 


\section{References}

(1) Kelly, A.; Macmillian, N. H. Strong Solids, 3rd ed. (Clarendon Press, Oxford, 1986).

(2) Liu, F.; Ming, P. B.; Li, J. Phys. Rev. B 2007, 76, 064120.

(3) Ogata, S.; Li, J.; Yip. S. Science 2002, 298, 807.

(4) Frenkel, J.Z. Phys. 1926, 37, 572.

(5) Orowan, E. Rep. Prog. Phys. 1949, 12, 185.

(6) Kumar, K. S.; Van Swygenhoven, H.; Suresh, S. Acta Mater. 2003, 51, 5743.

(7) Greer, J. R.; Nix, W. D. Phys. Rev. B 2006, 73, 245410.

(8) Roundy, D.; Krenn, C. R.; Cohen, M. L.; Morris, J. W. Jr. Phys. Rev. Lett. 1999, 82, 2713.

(9) Hall, E. O. Proc. Phys. Soc. London Ser. B 1951, 64, 747.

(10) Petch, N. J. J. Iron Steel Inst. 1953, 174, 25.

(11) Rupert, T. J.; Gianola, D. S.; Gan, Y.; Hemker, K. J. Science 2009, 326, 1686.

(12) Schiøtz, J.; Jacobsen, K. W. Science 2003, 301, 1357.

(13) Lu, K.; Lu, L.; Suresh, S. Science 2009, 324, 349.

(14) Callister, W. D. Fundamentals of Materials Science and Engineering, 2nd ed. (Wiley \& Sons., 2000).

(15) Wollmershauser, J. A.; Feigelson, B. N.; Gorzkowski, E. P.; Ellis, C. T.; Goswami, R.; Qadri, S. B.; Tischler, J. G.; Kub, F. J.; Everett, R. K. Acta Mater. 2014, 16, 9.

(16) Maglia, F.; Tredici I. G.; Anselmi-Tamburini, U. J Euro. Ceram. Soc. 2013, 33, 1045.

(17) Porter, D. A. Easterling, K. E. Phase Transformations in Metals and Alloys, 2nd ed. (Nelson Thornes, Cheltenham, U.K., 2000).

(18) Argon, A. S. Strengthening Mechanisms in Crystal Plasticity (Oxford University Press, Oxford, 2008). 
(19) Zhang, R. F.; Argon, A. S.; Veprek, S. Phys. Rev. B, 2010, 81, 245418.

(20) Lu, L.; Chen, X.; Huang, X.; Lu, K. Science 2009, 323, 607.

(21) Tian Y. J.; Xu, B.; Yu, D. L.; Ma, Y. M.; Wang, Y. B.; Jiang, Y. B.; Hu, W. T.; Tang, C. C.; Gao, Y. F.; Luo, K.; et al. Nature, 2013, 493, 385.

(22) Huang, Q.; Yu, D. L.; Xu, B.; Hu, W. T.; Ma, Y. M.; Wang, Y. B.; Zhao, Z. S.; Wen, B.; He, J. L.; Liu, Z. Y.; et al. Nature, 2014, 510, 250.

(23) Fujita, T.; Guan, P. F.; Reddy, K. M.; Hirata, A.; Guo, J. J.; Chen, M. W. Appl. Phys. Lett., 2014, 104, 021907.

(24) Domnich, V.; Reynaud, S.; Haber, R. A.; Chhowalla, M. J. Am. Ceram. Soc. 2011, $94,3605$.

(25) Bourne, N. K. Proc. R. Soc. Lond. A 2002, 458, 1999.

(26) Chen, M. W.; McCauley, J. W.; Hemker K. J. Science 2003, 299, 1563.

(27) Reddy, K. M.; Liu, P.; Hirata, A.; Fujita, T.; Chen, M. W. Nat. Comm. 2013, 4, 2483.

(28) An, Q.; Goddard, W. A. III; Cheng, T. Phys. Rev. Lett. 2014, 113, 095501.

(29) Farbaniec, L.; Hogan, J. D.; Xie, K. Y.; Shaeffer, M.; Hemker, K. J.; Ramesh, K. T. Int. J. Impact Eng. 2017, 99, 75 .

(30) Kresse, G.; Hafner, J. Phys. Rev. B 1993, 47, 558.

(31) Kresse, G.; Furthmuller, J. Comput. Mater. Sci. 1996, 6, 15.

(32) Kresse, G.; Furthmüller, J. Phys. Rev. B 1996, 16, 11169.

(33) Kresse, G.; Joubert, D. Phys. Rev. B 1999, 59, 1758.

(34) Roundy, D.; Krenn, C. R.; Cohen, M. L.; Morris, J. W., Jr. Phys. Rev. Lett. 1999, 82, 2713.

(35) Pan, Z. C.; Sun, H.; Zhang, Y.; Chen, C. F. Phys. Rev. Lett. 2009, 102, 055503.

(36) Pan, Z. C.; Sun, H.; Chen, C. F. Phys. Rev. Lett. 2007, 98, 135505. 
(37) Xie, K. Y.; An, Q.; McCauley, J.; Toksoy, F. M.; Haber, R. A.; Goddard, W. A. III; Hember, K. J. Phys. Rev. Lett. 2015, 115, 175501.

(38) Becke, A. D.; Edgecombe, K. E. J. Chem. Phys. 1990, 92, 5397.

(39) An, Q.; Goddard, W. A. III. Phys. Rev. Lett. 2015, 115, 105501.

(40) Xie, K. Y.; Kuwelkar, K.; Haber, R. A.; Lasalvia, J. C.; Hemker, K. J. J. Am. Ceram. Soc. 2016, 99, 1551. 


\section{Figure 1}
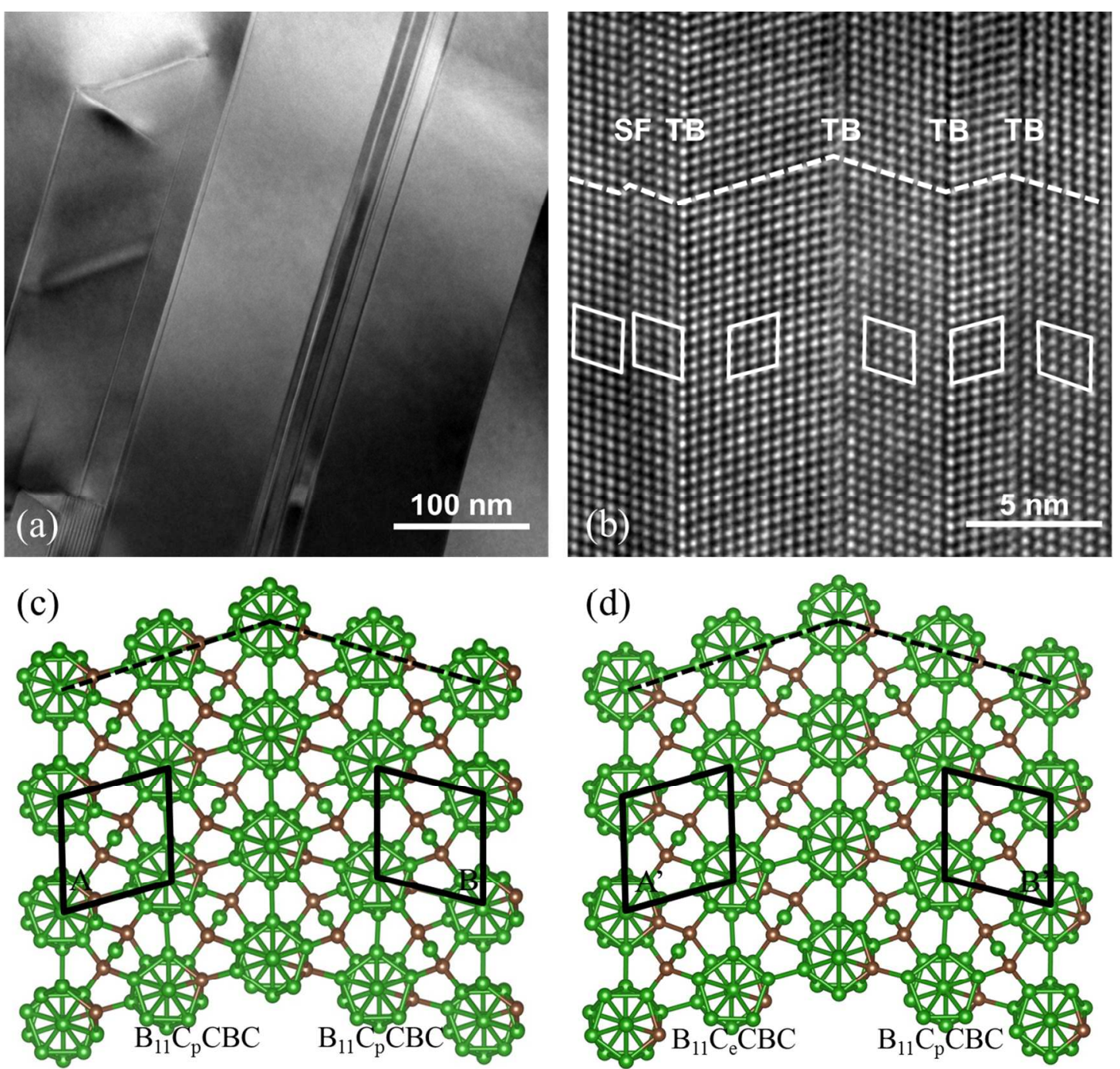

Figure 1. The nano-twinned $\mathrm{B}_{4} \mathrm{C}$ structures, where the boron and carbon atoms are represented by green and sienna balls, respectively. (a) TEM image showing the larger twin spacing at the 100 nanometer scale. (b) TEM image showing the fine scale twins with spacing of several nanometers. Here the symmetric twins have angles of $73.3^{\circ}$ while the asymmetric twins have angles of $72.3^{\circ}$ and $74.9^{\circ}$. (c) The DFT predicted symmetric twin, which leads to inclination angles of $73.1^{\circ}$ and $73.3^{\circ}$ on the two sides in excellent agreement with experiment. (d) The DFT predicted asymmetric twin exhibiting inclination angles of $72.9^{\circ}$ and $74.8^{\circ}$ on two sides, differing by $\sim 2^{\circ}$, just as in the experiments. 


\section{Figure 2.}
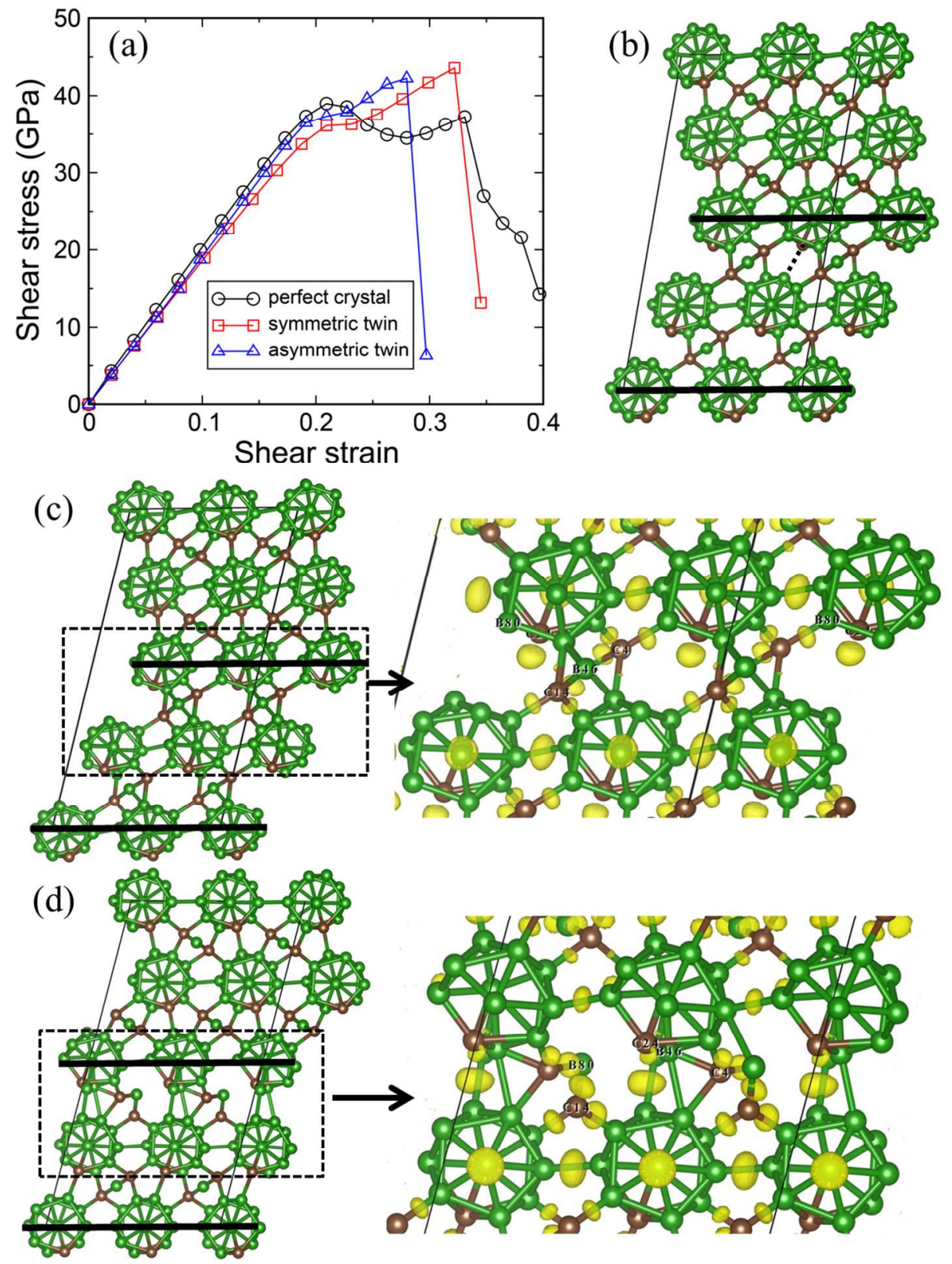

Figure 2. Shear-stress-shear-strain relationship and structural changes of nano-twinned $\mathrm{B}_{4} \mathrm{C}$. (a) The stress-strain relationship for the perfect crystal, the symmetric twin, and the asymmetric twin. (b) The structure of the symmetric nanotwin at 0.209 strain, the B-C bond between icosahedra in the lower half part starts to break. (c) The structure at 0.322 strain, reactive carbene character forms in the lower half part. (d) The structure at 0.345 strain showing that the $\mathrm{C}$-B-C chain (C4-B46-C14) reacts with the carbene (C24). This leads to breaking the C-B-C chain, inserting B46 into the icosahedron, and kicking out B80 from the icosahedron. The TB planes are represented by solid black line. The iso-surfaces from ELF analysis are displayed in yellow. 
Figure 3.
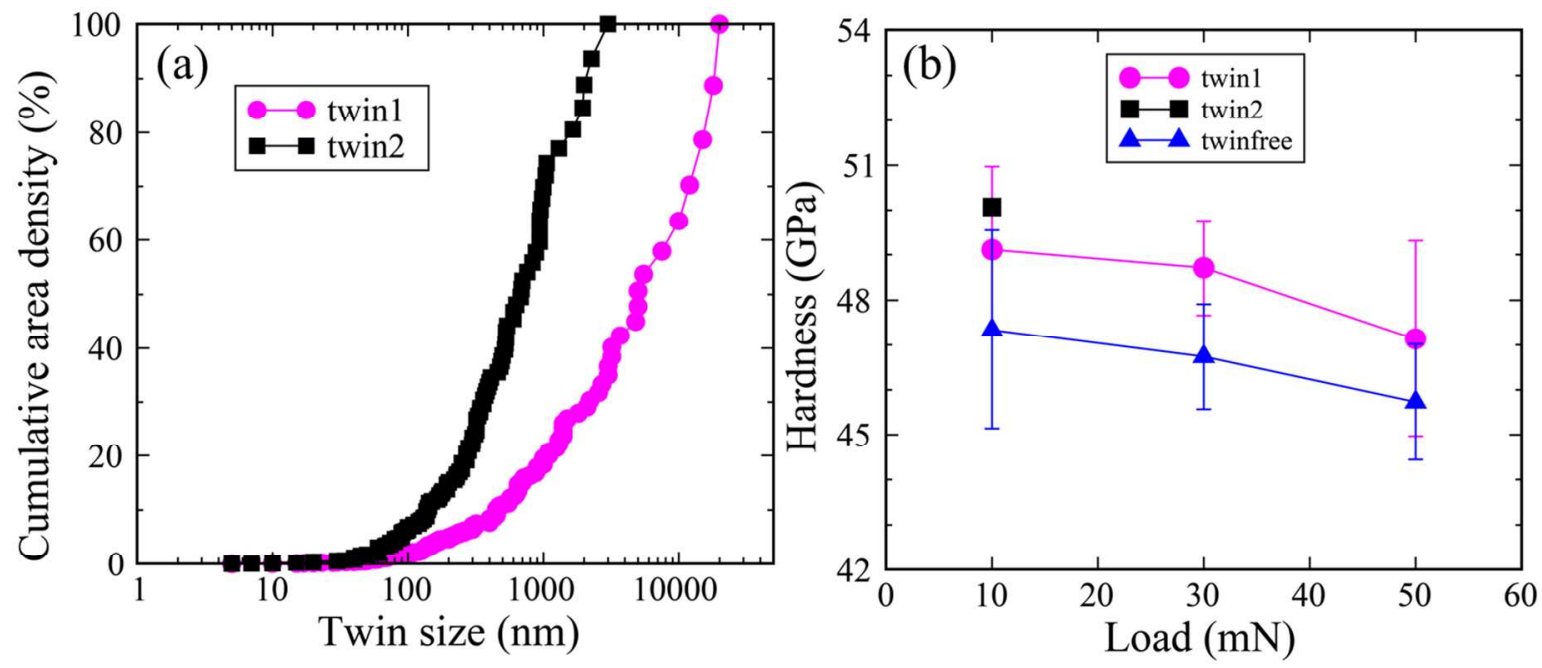

Figure 3. (a) Area-weighted cumulative distribution plots showing twin-2 sample has a higher twin density than twin-1 sample. (b) Experimental measurements of the hardness for two twinned and one twin-free samples. 


\section{Figure 4}

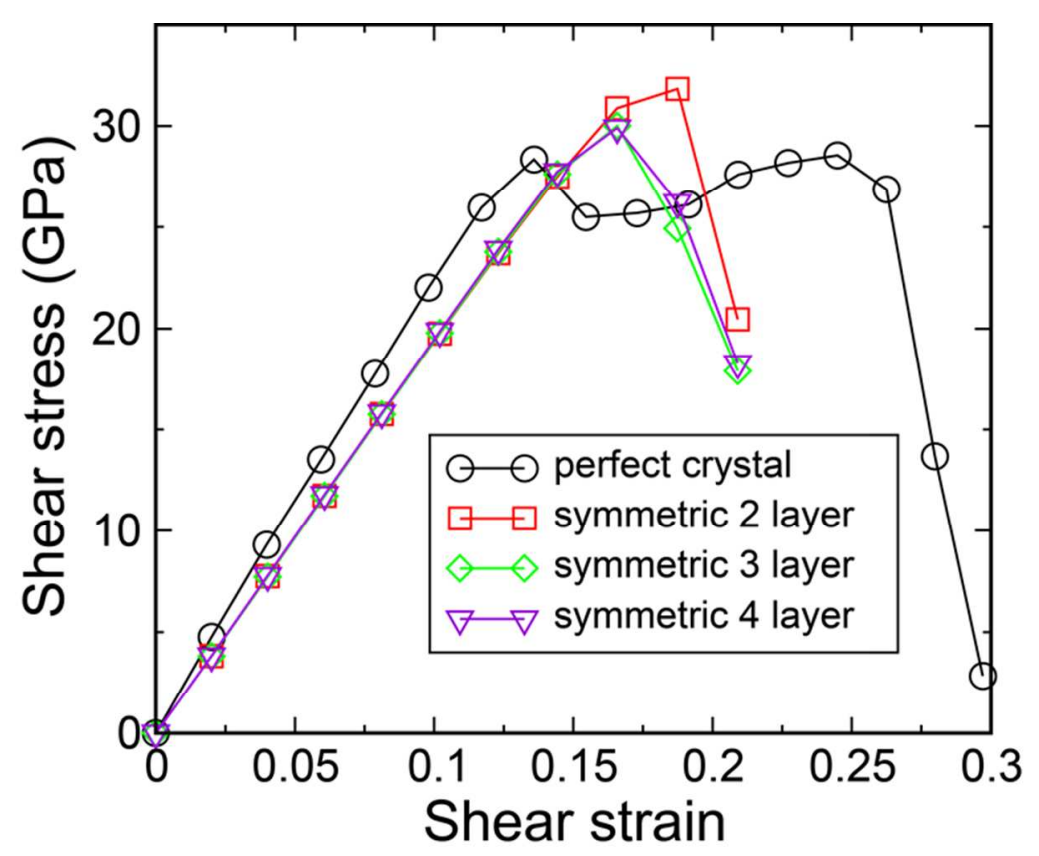

Figure 4. The $\mathrm{QM}$ stress-strain relationship for three nano-twinned structures and perfect $\mathrm{B}_{4} \mathrm{C}$ structures under biaxial shear deformations aimed at mimicking deformation under the indenter by imposing the relations $\sigma_{\mathrm{zz}}=\sigma_{\mathrm{zx}} \times \tan \Phi$ where $\sigma_{\mathrm{zz}}$ is the normal stress, $\sigma_{\mathrm{zx}}$ is the shear stress, and $\Phi$ is the centerline-to-face angle of the indenter. 


\section{Figure 5}
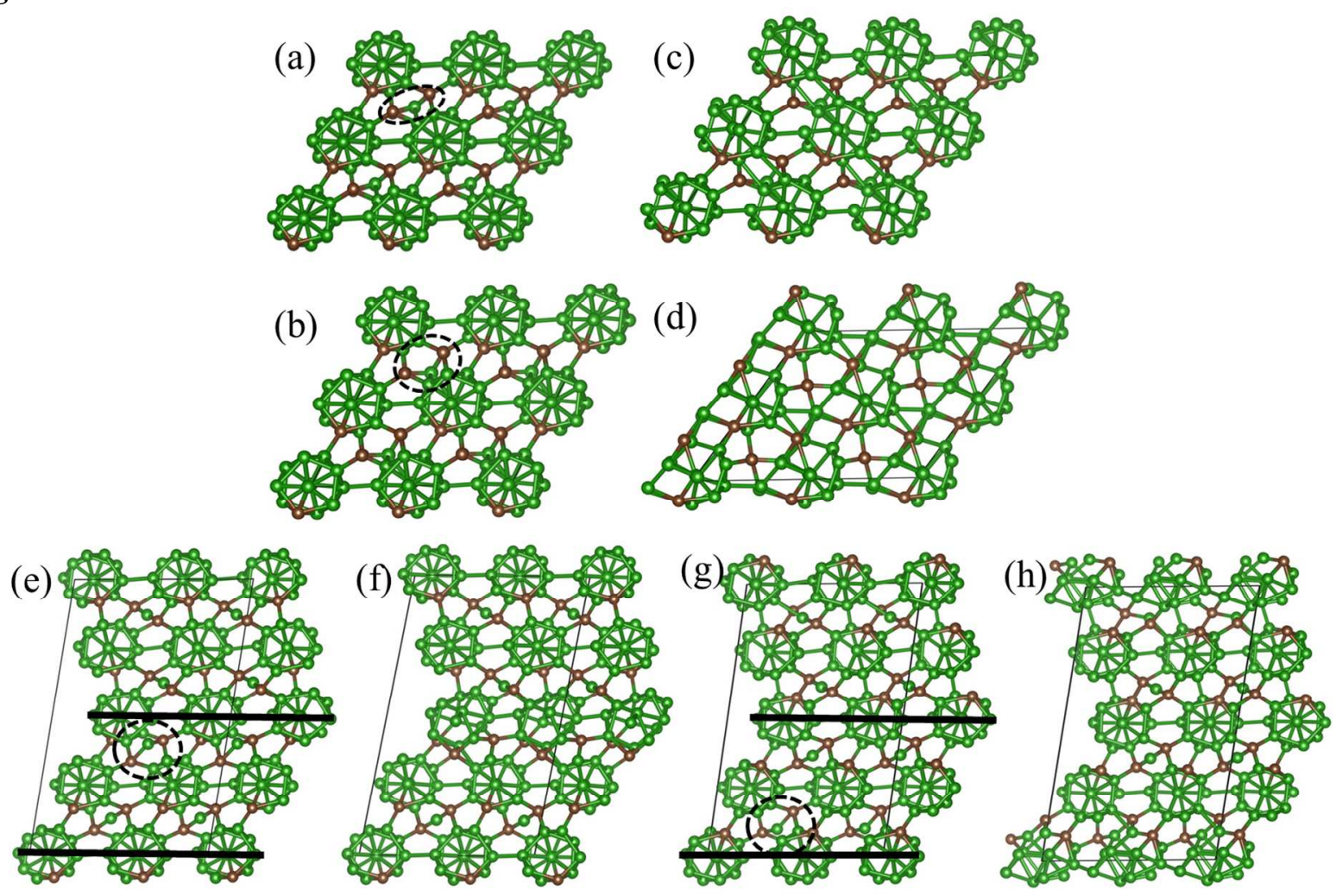

Figure 5. The structural changes for nano-twinned and perfect $\mathrm{B}_{4} \mathrm{C}$ structures under biaxial shear deformation aimed at mimicking deformation under the indenter. (a) Perfect $\mathrm{B}_{4} \mathrm{C}$ at 0.136 strain before plastic deformation. (b) Perfect $\mathrm{B}_{4} \mathrm{C}$ at 0.155 strain, where plastic deformation starts. (c) Perfect $\mathrm{B}_{4} \mathrm{C}$ at 0.263 strain, before failure. (d) Perfect $\mathrm{B}_{4} \mathrm{C}$ at 0.297 strain after failure. (e) Symmetric twin structure at 0.187 strain before failure. (f) Symmetric twin structure at 0.209 strain after failure. (g) Asymmetric twin structure at 0.173 strain before failure. (h) Asymmetric twin structure at 0.191 strain after failure. The TB planes are represented by the solid black line. The bent $\mathrm{C}-\mathrm{B}-\mathrm{C}$ chains are indicated with a dashed circle. The boron and carbon atoms are represented by the green and sienna balls, respectively. 